\title{
Ipriflavone Inhibits Bone Resorption in Intact and Ovariectomized Rats
}

\author{
M. G. Cecchini, H. Fleisch, R. C. Mühlbauer \\ Department of Pathophysiology, University of Berne, Murtenstrasse 35, CH-3010, Berne, Switzerland
}

\begin{abstract}
The aim of this study was to investigate the possible inhibitory effect of ipriflavone on bone resorption in rats. For this purpose, 10-week-old, intact and ovariectomized (OVX) rats, prelabeled from birth with $\left[{ }^{3} \mathrm{H}\right]-$ tetracycline, were used. Bone resorption was monitored by measuring the urinary excretion of $\left[{ }^{3} \mathrm{H}\right]$. The animals were fed a purified diet devoid of naturally occurring flavonoids. In the intact rats, the daily meal was given either as a single portion or divided into four portions, a procedure known to lead by itself to a decrease in bone resorption. Ipriflavone, given 7 days after OVX at the dose of $400 \mathrm{mg} / \mathrm{kg} \mathrm{B.W.} \mathrm{daily}$ mixed with the food, led within 2-3 days to a significant decrease in bone resorption equivalent to that of $27.2 \mu \mathrm{g} / \mathrm{kg}$ S.C. of $17 \beta$-estradiol. The inhibition was sustained for the length of the experiment, up to 21 days. Ipriflavone given 7 days before OVX prevented the increase in bone resorption induced by castration, the effect being dose-dependent between 50 and $400 \mathrm{mg} / \mathrm{kg} \mathrm{B.W}$. In contrast to $17 \beta$-estradiol, a 5-week treatment with ipriflavone failed to prevent the OVX-induced uterine atrophy. Significant inhibition of bone resorption was also seen in intact animals, provided they rapidly ingested the daily meal. Actually, the decrease in bone resorption induced by portioning the daily food masked the inhibitory effect of ipriflavone in intact animals. In conclusion, ipriflavone can decrease bone resorption in both intact and OVX animals given a purified diet as a single daily meal. In the OVX model, ipriflavone mimics the osteoprotective effect of estrogen. However, the lack of a uterotropic effect suggests that the compound can discriminate between bone and reproductive tissues.
\end{abstract}

Key words: Ipriflavone - Bone resorption - Ovariectomized rats.

Ipriflavone is a synthetic derivative of a natural isoflavone. First clinical trials indicate that the compound exerts a beneficial effect in osteopenic diseases [1-3]. It is not clear yet whether ipriflavone acts primarily by inhibiting bone resorption or by stimulating bone formation. Inhibition of osteoclast recruitment in both in vitro $[4,5]$ and in vivo [6], and induction of markers of the osteoblast phenotype in vitro $[7,8]$ have been demonstrated. However, in the rat model of osteopenia following ovariectomy [9], ipriflavone did not reduce bone resorption when administered alone, but it did enhance the therapeutic effects of estrogen. Confounding factors may explain the lack of the protective ef-

Correspondence to: $\mathrm{M} . \mathrm{G}$. Cecchini fect of ipriflavone per se on bone loss due to estrogen withdrawal in animals. Therefore, we investigated in vivo the modalities by which an intrinsic inhibitory activity of ipriflavone on bone resorption can be disclosed.

For measuring bone resorption, we used a technique based on long-term prelabeling with $\left[{ }^{3} \mathrm{H}\right]$-tetracycline and the subsequent determination of urinary excretion of the tracer (Fig. 1). This technique allows monitoring of bone resorption, daily or at shorter intervals, over extended periods of time [10].

According to the components used, rat chow may contain large amounts of naturally occurring flavonoids. In order to exclude substances that might interfere with the effect of ipriflavone, and to mimic a typical human "western diet" with large proportions of refined components, we have used a "purified" diet that contains casein as the protein source and starch as the carbohydrate source. In rats fed this diet, ipriflavone significantly reduced bone resorption induced by ovariectomy.

Surprisingly, ipriflavone given to intact rats failed to show any appreciable effect on bone resorption. However, careful recording of the feeding habit revealed that intact animals given ipriflavone mixed with the food ingested their meals at a slower rate than control rats.

Bone resorption in the rat displays a diurnal rhythm that is blunted by food fractionation [11]. Therefore, we have compared the effect of ipriflavone on intact animals ingesting the daily food either as a single meal or fractionated into four portions. We found that ipriflavone was inhibiting bone resorption only in rats that were rapidly ingesting their daily food as a single meal.

\section{Material and Methods}

Animals

At the age of 7 weeks, Wistar rats from our own breeding colony were housed in individual metabolic cages. Until the experiments, all animals had free access to tap water and were fed a standard laboratory chow ad libitum (Kliba 331; Klingentalmühle, Kaiseraugst, Switzerland), containing $1.0 \mathrm{~g} \mathrm{Ca}, 0.7 \mathrm{~g} \mathrm{P}$ and $80 \mathrm{IU}$ vitamin- $\mathrm{D}_{3} / 100 \mathrm{~g}$ dry weight. During the experiments, some groups continued to receive lab chow, $14 \mathrm{~g}$ dry weight per day. The other groups were switched to the purified diet (see introduction), 14.7 $\mathrm{g}$ dry weight per day, containing $1.1 \mathrm{~g} \mathrm{Ca}$ and $1.2 \mathrm{~g} \mathrm{P} / 100 \mathrm{~g}$ dry weight.

\section{$\left[{ }^{3} H\right]$-tetracycline Labeling}

Beginning at the age of 2-3 days, the rats were injected twice a week for 6 weeks with increasing amounts of a solution containing 


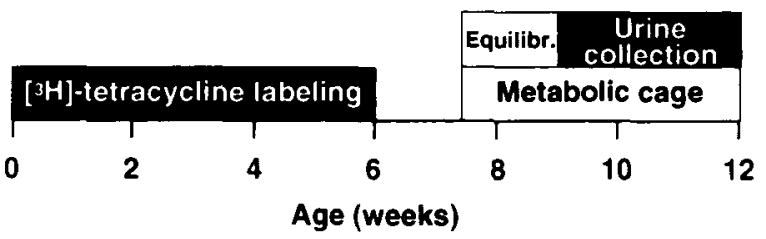

Fig. 1. Schematic representation of the experimental protocol used for monitoring bone resorption in rats with the urinary excretion of $\left[{ }^{3} \mathrm{H}\right]$-tetracycline.

$10 \mu \mathrm{Ci} / \mathrm{ml}$ of $\left[{ }^{3} \mathrm{H}\right]$-tetracycline $\left(\left[{ }^{3} \mathrm{H}\right]-\mathrm{TC}\right)$ (New England Nuclear, Boston, MA) dissolved in $0.15 \mathrm{M} \mathrm{NaCl}$, as described previously [10].

\section{Feeding Schedule}

Wet food (26 g) corresponding to $14.7 \mathrm{~g}$ of dry matter, was provided at 11 A.M. In the experiment with food fractionation the rats received $24 \mathrm{~g}$ of wet food, corresponding to $13.5 \mathrm{~g}$ of dry matter, provided either as a single meal at 11 A.M., or in four portions of 6 g each, every 6 hours. Before the experiment started, all animals were trained to ingest the entire amount of food within 2 hours. In all cases the animals had free access to tap water.

\section{Urine Collection}

After a 7-10-day adaptation, the urine was collected every 24 hours and the $\left[{ }^{3} \mathrm{H}\right]$ content was determined by liquid scintillation spectrometry, as previously described [10]. After an initial 5-7 day monitoring of the baseline urinary $\left[{ }^{3} \mathrm{H}\right]$-excretion, the animals were randomly allocated to treatment groups.

\section{Ovariectomy}

For some experiments, the rats, aged 9-9.5 weeks, were either bilaterally ovariectomized (OVX) or sham-operated (SHAM) under ether anesthesia. In the SHAM rats, the ovaries were bilaterally exposed and handled, but not removed. To verify complete removal of the ovaries, at the end of the experimental period the uterus was excised and the weight was determined.

\section{Treatment}

Ipriflavone was carefully mixed with the food, as dry substance, at daily doses of 400,150 , and $50 \mathrm{mg} / \mathrm{kg} \mathrm{B}$.W. When the experimental protocol included OVX, the compound was administered either 7 days before or 7 days after the surgical intervention.

$17 \beta$-estradiol (Sigma, St. Louis, MO), dissolved in corn oil, was administered subcutaneously to OVX rats, beginning either on the day of surgery or 7 days after, at the daily dose of $27.2 \mu \mathrm{g} / \mathrm{kg}$ B.W. immediately before the 11 A.M. meal.

Treatment with both ipriflavone and $17 \beta$-estradiol was maintained until the end of each experiment.

\section{Statistics}

Statistical differences between experimental groups were assessed by one-way analysis of variance, followed by the posthoc Newman-Keuls multiple comparison test. A $P$ value of less than 0.05 was considered significant.
Results

\section{Effect of Ipriflavone on Bone Resorption in OVX Rats}

First we investigated whether ipriflavone could prevent or arrest the OVX-induced bone resorption. Within 1 week from castration bone resorption in OVX rats significantly increased, attaining an average of $40 \%$ increase in bone resorption as compared with SHAM animals. 17 $\beta$-estradiol, administered daily starting on the day of OVX, maintained bone resorption at the same level of SHAM $(P<0.01$ versus OVX). Ipriflavone, administered to OVX rats starting 7 days before or after castration at the daily dose of 400 $\mathrm{mg} / \mathrm{kg} \mathrm{B.W}$., was equally effective as $17 \beta$-estradiol in reducing OVX-induced bone resorption to the values of SHAM animals $(P<0.001$ or $P<0.01$ versus OVX when given from day -7 or day +7 , respectively). In addition, there was a slight inhibition of basal bone resorption already during the period of administration prior to castration.

Next, we investigated whether the inhibitory effect of ipriflavone on OVX-induced bone resorption was dose dependent. The various daily doses of ipriflavone were administered and mixed with food beginning 7 days before OVX to rats fed with purified diet. Doses of 50 and 150 $\mathrm{mg} / \mathrm{kg} \mathrm{B}$.W. reduced bone resorption significantly $(P<0.01$ and $P<0.05$ versus $\mathrm{OVX}$, respectively), both attaining an average of $50 \%$ of the inhibitory effect exerted either by $17 \beta$-estradiol or ipriflavone, $400 \mathrm{mg} / \mathrm{kg} \mathrm{B.W}$., given in the same experiment. The uterine weight of OVX rats was not significantly affected by ipriflavone at any of the doses administered. In contrast, the uterine weight of the OVX animals that received $17 \beta$-estradiol did not differ significantly from that of the SHAM animals.

\section{Effect of Ipriflavone on Bone Resorption in Intact Rats}

When we investigated the effect of ipriflavone in intact rats fed with the purified diet, the compound was unable to significantly influence basal bone resorption. However, careful recording of the feeding habit revealed that intact animals given ipriflavone mixed with the food ingested the meal at a slower rate than control rats. Fractionation of daily food intake by itself decreases bone resorption [11] and this mechanism may have masked the inhibitory effect of ipriflavone on bone resorption in intact animals eating slowly. In contrast, OVX animals, known to be prone to increased appetite, did not present relevant modifications of their feeding habit when treated with ipriflavone. This may explain the discrepancy in effect of ipriflavone between intact and OVX rats.

A subsequent experiment showed that indeed ipriflavone, at the daily dose of $400 \mathrm{mg} / \mathrm{kg} \mathrm{B.W.,} \mathrm{significantly}$ inhibited $(P<0.01)$ basal bone resorption in intact rats that were previously trained to rapidly ingest the daily food as a single portion. In contrast, ipriflavone, at the same dose, failed to reduce bone resorption when administered to intact rats that consumed the daily food fractionated into four portions.

\section{Discussion}

In this study we demonstrate that ipriflavone per se inhibits bone resorption, both in intact and OVX rats that are fed a purified diet that is likely to be devoid of flavonoids and 
other compounds that mimic estrogen effects on some target tissues. In the OVX rats the magnitude of the effect of a daily dose of $400 \mathrm{mg} / \mathrm{kg} \mathrm{B}$.W. of ipriflavone is equivalent to that of $17 \beta$-estradiol at the daily dose of $27.2 \mu \mathrm{g} / \mathrm{kg} \mathrm{B} . \mathrm{W}$. Furthermore, we show that the effect of ipriflavone is no longer evident when the daily food is fractionated, a procedure that by itself decreases bone resorption [11].

It has previously been reported that ipriflavone, when administered alone, could not prevent bone loss in the OVX rat [9]. The fact cannot be excluded that in that study, natural flavonoids contained in the "commercial diet" used or slow food consumption may have masked the effect of ipriflavone.

It is not known whether similar confounding factors may be relevant in the human species. Ipriflavone per se has been shown to exert beneficial effects on the osteopenia induced in women by either iatrogenic [2] or physiological estrogen deficiency [1, 3]. Nevertheless, it is well documented that dietary flavonoids alter the hormonal status of premenopausal women [12]. Accordingly, the possibility that the diet composition and/or meal frequency may play a role in the clinical evaluation of the therapeutic effect of ipriflavone should be taken into account.

Recently, it has been elucidated in vitro that neither ipriflavone nor the majority of its metabolites interact directly with classical type I estrogen receptors [13]. However, ipriflavone and its biologically most active metabolite III bind to nuclear receptors that are likely to be part of the steroid receptor superfamily [13]. Consistent with these findings in vitro, it has been reported previously that ipriflavone by itself is devoid of any estrogenic effect in both OVX animals [9, 14] and in postmenopausal women [15]. Our present investigation confirms that the compound does not exert uterotropic effects in OVX animals.

The evidence stated above, indicating that ipriflavone counteracts bone loss due to estrogen deficiency while not exerting an uterotropic effect, suggests that this compound may behave as a selective estrogen receptor modulator (SERM). Other compounds, such as raloxifene [16] and droloxifene [17], with the characteristics of the SERMs, have been proposed as pharmacological inhibitors of postmenopausal bone loss. However, in contrast to ipriflavone, these two compounds have been shown to interact with classical estrogen receptors $[18,19]$ and exert a slight but significant uterotropic effect in OVX rats [16, 17]. Thus, ipriflavone may represent a class of compounds that mimic the protective effect of estrogen on bone tissue by a mechanism that seems to involve a transduction pathway different from that of estrogen. Interestingly, a diet rich in flavonoids has been shown to reduce serum cholesterol [12]. Further investigations are warranted in order to verify whether ipriflavone exerts a protective effect also against the increased risk of developing ischemic heart disease characteristic of postmenopausal women.

While clearly demonstrating that ipriflavone can inhibit bone resorption in the rat, the present study also suggests that confounding factors such as diet formulation and/or feeding habit should be taken into consideration when investigating the protective effect against bone loss of new compounds in experimental animals.

Acknowledgments. We thank I. Tschudi and U. Mäusli for skillful technical assistance and I. Ryf for revising the English typescript. This investigation was supported by Chiesi Farmaceutici SpA, Parma, Italy.

\section{References}

1. Agnusdei D, Camporeale A, Zacchei F, Gennari C, Baroni MC, Costi D, Biondi M, Passeri M, Ciacca A, Sbrenna C, Falsettini E, Ventura A (1992) Effects of ipriflavone on bone mass and bone remodeling in patients with established postmenopausal osteoporosis. Curr Ther Res 51:82-91

2. Gambacciani M, Spinetti A, Cappagli B, Taponeco F, Felipetto R, Parrini D, Cappelli N, Fioretti P (1993) Effects of ipriflavone administration on bone mass and metabolism in ovariectomized women. J Endocrinol Invest 16:333-337

3. Valente M, Bufalino L, Castiglione GN, D'Angelo R, Mancuso A, Galoppi P, Zichella L (1994) Effect of 1-year treatment with ipriflavone on bone in postmenopausal women with low bone mass. Calcif Tissue Int 54:377-380

4. Notoya K, Yoshida K, Taketomi S, Yamazaki I, Kumegawa M (1992) Inhibitory effect of ipriflavone on pit formation in mouse unfractionated bone cells. Calcif Tissue Int 51:S3-S6

5. Morita I, Sakagushi K, Kurachi T, Murota S (1992) Ipriflavone inhibits murine osteoclast formation in vitro. Calcif Tissue Int 51:S7-S10

6. Bonucci E, Ballanti P, Martelli A, Mereto E, Brambilla G, Bianco P, Bufalino L (1992) Ipriflavone inhibits osteoclast differentiation in parathyroid transplanted parietal bone of rats. Calcif Tissue Int 50:314-319

7. Benvenuti S, Tanini A, Frediani U, Bianchi S, Masi L, Casano R, Bufalino L, Serio M, Brandi ML (1991) Effects of ipriflavone and its metabolites on a clonal osteoblastic cell line. $J$ Bone Miner Res 6:987-996

8. Notoya K, Yoshida K, Tsukuda R, Taketomi S (1994) Effect of ipriflavone on expression markers characteristic of the osteoblast phenotype in rat bone marrow stromal cell culture. $\mathbf{J}$ Bone Miner Res 9:395-400

9. Yamazaki I, Shino A, Tsukuda R (1986) Effect of ipriflavone on osteoporosis induced by ovariectomy in rats. J Bone Miner Metab 3:205-210

10. Mühlbauer RC, Fleisch H (1990) A method for continual monitoring of bone resorption in rats: evidence for a diurnal rhythm. Am J Physiol 259:R679-R689

11. Mühlbauer RC, Fleisch H (1995) The diurnal rhythm of bone resorption in the rat. Effect of feeding habits and pharmacological inhibitors. J Clin Invest 95:1933-1940

12. Cassidy A, Bingham S, Setchell KDR (1994) Biological effects of a diet of soy protein rich in isoflavones on the menstrual cycle of premenopausal women. Am J Clin Nutr 60 : 333-340

13. Petilli M, Fiorelli G, Benvenuti S, Frediani F, Gori F, Brandi ML (1995) Interactions between ipriflavone and the estrogen receptor. Calcif Tissue Int 56:160-165

14. Yamazaki I (1986) Effect of ipriflavone on the response of uterus and thyroid to estrogen. Life Sci 38:757-764

15. Melis GB, Paoletti AM, Cagnacci A, Bufalino L, Spinetti A, Gambacciani M, Fioretti P (1992) Lack of any estrogenic effect of ipriflavone in postmenopausal women. $\mathbf{J}$ Endocrinol Invest 15:755-761

16. Black LJ, Sato M, Rowley ER, Magee DE, Bekele A, Williams DC, Cullinan GJ, Bendele R, Kauffman RF, Bensch WR, Frolik CA, Termine JD, Bryant HU (1994) Raloxifene $(\mathrm{LY} 139481 \mathrm{HCl})$ prevents bone loss and reduces serum cholesterol without causing uterine hypertrophy in ovariectomized rats. J Clin Invest 93:63-69

17. Ke HZ, Simmons HA, Pirie CM, Crawford DT, Thompson DD (1995) Droloxifene, a new estrogen antagonist/agonist, prevents bone loss in ovariectomized rats. Endocrinology 136: 2435-2441

18. Black LJ, Jones CD, Falcone JF (1983) Antagonism of estrogen action with a new benzothiophene-derived antiestrogen. Life Sci 32:1031-1036

19. Eppenberger U, Wosikowski K, Kung W (1991) Pharmacologic and biologic properties of droloxifene, a new antiestrogen. Am J Clin Oncol (suppl 2) 14:S5-S14 